\title{
Il pessimismo mitteleuropeo: Otto Weininger ${ }^{1}$
}

\author{
Fabio Ciracì \\ Dottore in Filosofia; Professore nell'Università del Salento (Lecce-Itália) \\ Segretario del "Centro Interdipartimentale di ricerca su Arthur Schopenhauer e la sua scuola"
}

RIASSUNTO: Con il presente contributo si intende contestualizzare l'opera e il pensiero del filosofo viennese Otto Weininger, collocandolo all'interno della cultura mitteleuropea. Si vuole, quindi, esporre brevemente la formazione del pensatore austriaco in relazione alla cultura pessimistica della cosiddetta finis Austriae. Inoltre, si vogliono mettere in luce gli aspetti filosofici fondamentali della sua metafisica dell'amor sessuale, indicandone le fonti scientifiche e letterarie che lo hanno ispirato, per rilevare la tensione morale e religiosa del pensiero filosofico weiningeriano, in base alla quale è possibile parlare di un pessimismo mistico, edulcorato dalla prospettiva escatologica. In conclusione, si vuol confrontare il pensiero di Weininger con quello di Arthur Schopenhauer e Friedrich Nietzsche, mettendone in rilievo i punti di distanza e di diversità con i due filosofi.

PAROLE-CHIAVI: Weininger; pessimismo; amor sessuale.

RESUMO: Com o presente estudo pretende-se contextualizar a obra e o pensamento do filósofo vienense Otto Weininger, tomando-o no interior da cultura centro-européia. Almeja-se, assim, expor brevemente a formação do pensador austríaco em relação à cultura pessimista da assim chamada finis Austriae. Além disso, pretende-se trazer à luz os aspectos filosóficos fundamentais da sua metafísica do amor sexual, indicando as fontes científicas e literárias que o inspiraram a fim de detectar a tensão moral e religiosa do pensamento filosófico weiningeriano, com base na qual é possível falar de um pessimismo místico, recheado pela prospectiva escatológica. Por fim, objetiva-se confrontar o pensamento de Weininger com aqueles de Arthur Schopenhauer e de Friedrich Nietzsche, destacando os pontos de distância e de diversidade em relação aos dois filósofos.

PALAVRAS-CHAVE: Weininger; pessimismo; amor sexual.

\section{Il contesto}

La ricostruzione del contesto storico della Vienna fin-de-siècle è indispensabile per descrivere e connotare il pessimismo viennese dell'ultima Jahrhundertwende. Ci riferiamo in particolare alla "giovane Vienna", che si era formata con la musica di Wagner e di Beethoven, che aveva letto il Kant della ragion pratica alla luce del pessimismo metafisico di Schopenhauer, e che riscopriva il vitalismo tragico di Nietzsche. A questa Vienna

\footnotetext{
${ }^{1}$ Il presente saggio è stato da me presentato in qualità di relatore al XXXI Seminario Internazionale di Studi italo-tedeschi sul tema Mito e realtà dell'impero austroungarico, organizzato dall'Accademia di Studi Italo-Tedeschi di Merano in collaborazione con le Università del Salento e di Friburgo i. B., tenutosi a Merano dal 27 al 29 aprile 2009.
} 
appartenevano personalità come Karl Kraus (1874-1936), Hugo von Hofmannsthal (1874-1929), Stefan Zweig (1881-1942) e Arthur Schnitzler (1862-1931), tutti frequentatori del celebre Caffé Griensteidl.

Questi intellettuali si riconoscevano in un medesimo sentire, riconoscevano nell'esistenza il mal de vivre, inteso come malattia (Svevo e Michelstaedter), come impossibilità della comunicazione (Hofmannsthal), oppure come avvento del nichilismo (Kafka, Musil), cercando ognuno la propria via di redenzione attraverso l'arte e la filosofia. Un'esperienza intellettuale, quella viennese, non riducibile ad un unico paradigma, ma piuttosto ascrivibile ad una Zeitstimmung unitaria.

A costruire questa immagine del mondo disincantata e a porre le basi per l'analisi della cosiddetta Krisis aveva contribuito fortemente l'introduzione del metodo psicoanalitico di Sigmund Freud, che gli intellettuali della finis Austriae intendevano utilizzare esclusivamente come strumento di diagnosi, senza prefigurare però alcuna prognosi o cura della malattia, che in questa cultura assume il ruolo di vero e proprio principium individuationis dell'uomo, di tratto connotativo dell'individualità. Ma alla scoperta dell'inconscio, come l'ha definita Hellenberger ${ }^{2}$, si univa anche la divulgazione della filosofia schopenhaueriana e di quella nietzscheana ${ }^{3}$. Un ruolo a sé, invece, assumeva l'interpretazione della ragion pratica di Kant, letta attraverso la lente di un certo titanismo tardo-romantico, in cui la cieca volontà schopenhaueriana veniva tradotta nella volontà buona di kantiana memoria. Questo è il caso, per esempio, di Theodor Lessing, che a partire dalla lezione di Goethe e di Nietzsche, aveva introdotto il concetto di Sinngebung, ovvero di dazione di senso individuale, nel tentativo di superare il nichilismo profetizzato dall'autore dello Zarathustra. Contro questa tendenza moralistica, dalle tinte fichtiane ${ }^{4}$, si

\footnotetext{
${ }^{2}$ Si confronti Henri F. Hellenberger, The Discovery of the Unconscious. The History and Evolution of Dynamic Psychiatry, Basic Books, New York 1970, tr. It. Id., La scoperta dell'inconscio. Storia della psichiatria dinamica, 2 voll., Bollati Boringhieri, Torino 2001, in particolare vol. II, pp. 961-969.

${ }^{3}$ Id., pp. 626-7: «Si ricordi che la filosofia di Schopenhauer era, in ampia misura, un tipo di misticismo sessuale fra tanti altri. Due esponenti successivi di tale tendenza conobbero Freud: Wilhelm Fliess e Otto Weininger. [...] Il misticismo sessuale che pervadeva l'atmosfera intellettuale a Vienna alla fine del diciannovesimo secolo e all'inizio del ventesimo si estese anche alla nuova scienza della patologia sessuale».

${ }^{4}$ L'elemento morale fichtiano è largamente presente nella metafisica weiningeriana, come si evince dalla lettura della sua opera principale, Sesso e carattere (d'ora in poi $\mathrm{GuCh}$ ), tr. it. di J. Evola, intr. di F. Antonini, coll. Orizzonti dello spirito, Edizioni Mediterranee, Roma, 1992. Si veda, per esempio, il lungo capitolo VII «La logica, l'etica e l'Io», in cui Weininger rielabora la distinzione kantiana fra io empirico e io intelligibile, cercando di conciliarla con l'autotesi dell'Io di Fichte. GuCh, p. 206 e ss.
} 
muovevano autori come Svevo e Musil, i quali prendevano atto della crisi del sistema dei valori dei ceti borghesi e ne analizzavano lucidamente gli aspetti psicologici e sociali.

Inoltre, la Krisis aveva depotenziato ed eroso anche il linguaggio, sia quello logico-discorsivo che quello simbolico-poetico, e non era certo un caso che la questione del potere significativo del linguaggio giungesse sino alla Sprachkritik del viennese Ludwig Wittgenstein (1889-1905), come eredità della cultura mitteleuropea di questo periodo 5 .

Si tratta, allora, di una mitteleuropea intesa non solo in senso geopolitico, ma anche nel senso di una nuova Weltanschauung, di quello specifico mitteleuropeo, come lo ha denominato Camerino $^{6}$, in cui ricorrono i temi paradigmatici del centro-periferia dell'impero, del rapporto padre-figli, della Sprachkritik, della centralità della malattia, ecc. Il pessimismo mitteleuropeo assume, quindi, una sua componente psicologica e una sua dimensione esistenziale. Esso si può collocare temporalmente fra la Lettera di Lord Chandos (1902) di von Hofmannsthal, Sesso e carattere (1903) di Weininger, La persuasione e la rettorica (1913) di Michelstaedter, la Coscienza di Zeno (1919-23) di Svevo, e L'uomo senza qualità (1930-33) di Musil negli anni Trenta.

In questa cornice, la comunità ebraica viennese si era amalgamata alla società civile della capitale asburgica e dopo la cosiddetta diaspora verso Vienna, inaugurata da Francesco Giuseppe II con il suo discorso An meiner Völker, la componente ebraica aveva assunto un ruolo significativo nell'economia e nella cultura viennese. Al primo periodo d'integrazione dei padri, era poi seguito quello della dissoluzione e del disagio dei figli, che rifiutavano una tradizione ormai consolidata, svuotata nel contenuto dall'abitudine dei riti. Cosí, opponendosi alla vuota formalità delle prassi sociali e dei cerimoniali religiosi, come per riflesso, questa seconda generazione di origine ebraica in terra d'Austria si opponeva al contempo anche alla vuota routine della macchina burocratica, in cui si rifletteva il pesante apparato statale asburgico. Questa critica dei processi sociali, infatti, finiva per generalizzarsi da condizione individuale a stato esistenziale collettivo, sino ad assurgere a categoria ermeneutica del disagio esistenziale dell'uomo moderno.

\footnotetext{
${ }^{5}$ Cfr. A. Janik e S. Toulmin, La grande Vienna. Nella Vienna di Schönberg, di Musil, di Kokoschka e del dottor Freud, la formazione intellettuale del grande filosofo Wittgenstein, Garzanti, 1975; in particolare il cap. «Linguaggio, etica e raffigurazione», pp. 121-168.

${ }^{6}$ A. G. Camerino, A mo' di conclusione. Lo specifico mitteleuropeo e i maggiori scrittori giuliani del Novecento, in Id., La persuasione e i simboli: Michelstaedter e Slataper, Liguori, Napoli 2005, p. 107 e ss.
} 
Se è necessario parlare della "storia dell'assimilazione"” del popolo ebraico, in taluni casi però, come in quello di Weininger, l'impossibilità di accettare l'integrazione si tramutò inconsciamente in quello che Theodor Lessing avrebbe poi definito come lo jüdischer Selbsthaß $\beta^{8}$, l'odio di sé ebraico. All'interno di questo quadro storico di riferimento si svolge la vicenda intellettuale di Otto Weininger.

\section{La formazione e l'opera di Weininger}

Otto Weininger nasce a Vienna il 3 aprile del 1880, da una numerosa famiglia di origine ebraica". Dal padre riceve una buona educazione musicale, in cui primeggia "il culto di Wagner"10 e durante gli anni scolastici si forma alle lettere, studiando storia, filosofia e soprattutto filologia, manifestando inoltre una particolare inclinazione per le lingue: padroneggia il francese, l'inglese e l'italiano, il norvegese, lo spagnolo, il latino e il greco. Weininger completa gli studi all'università di Vienna, che frequenta dal 1898 al 1900, e si sostiene impartendo lezioni private, grazie alle quali stringe numerose amicizie, dando inoltre prova di un indiscusso carisma sui suoi coetanei.

È negli anni dell’Università che il suo interesse si allarga dalle scienze dello spirito a quelle della natura, in particolare alla matematica, alla fisica e alle scienze naturali. Legge soprattutto i testi di autori appartenenti alla cosiddetta Naturphilosophie di ispirazione schellinghiana, come Gustav Fechner e Rudolf Hermann Lotze, dai quali è profondamente colpito. Inoltre, viene in contatto con l'ambiente viennese del positivismo scientifico e subisce l'influsso di R. Avenarius, di cui legge La critica dell'esperienza pura. Ma l'irrequietudine che caratterizzerà l'esistenza di Weininger lo costringe spesso a mutare il suo intereresse, e probabilmente questo atteggiamento è all'origine del suo dilettantismo scientifico e filosofico.

\footnotetext{
${ }^{7}$ G. A. Camerino, La persuasione e i simboli..., cit., p. 114.

${ }^{8}$ Th. Lessing, Der jüdische Selbsthaß (1930). Mit einem Essay von Boris Groys, Nachdr. der Ausg. Matthes \& Seitz, Berlin 2004.

${ }^{9}$ Per la ricostruzione della vicenda biografica di Weininger, si rimanda all'ormai classico studio del curatore dei Werke di Weininger, J. Le Rider, Le cas O. Weininger. Racines de l'antiféminisme et de l'antisémitisme, Presses Universitaires de France, Paris 1982, poi ampliato nell'edizione tedesca Der Fall O. Weininger: Wurzeln des Antifeminismus und Antisemitismus, Löcker, Wien-München 1985.

${ }^{10}$ Weininger scriverà: «Wagner, il piú grande spirito dopo Cristo», in GuCh, p. 430.
} 
A partire dal 1900, si dedica sempre piú alla biologia e alla medicina, ed infine si concentra sulla teoria della sessualità, tanto da decidere di svolgere la sua tesi di laurea sul tema Eros e Psyche, in cui è manifesto anche l'influsso delle teorie empirio-criticiste di E. Mach (1838-1916), che Weininger segue a lezione. Teorie, che però in seguito Weininger abbandonerà quasi del tutto. È in questo periodo che formula, per la prima volta, la celebre legge di attrazione sessuale, che convoglierà poi nel terzo capitolo di Geschlecht und Charakter. Per approfondire le sue ricerche universitarie, si ritira in solitudine e si dedica a tempo pieno all'approfondimento del problema della sessualità e dell'emancipazione della donna, unendo agli studi naturalistici la lettura di testi religiosi, come gli scritti di Tertulliano e di Agostino, ma anche di Platone e di Plotino. E sarà proprio sulla base di queste ultime letture che Weininger tenterà di dare una fondazione metafisica e mistica al proprio sistema.

Sempre nello stesso anno si laurea e poco dopo decide di abiurare la sua origine ebraica, battezzandosi con rito protestante. In questo periodo si sviluppa un profondo sentimento di disagio per la sua situazione esistenziale, quella di ebreo antisemita, che lo condurrà poi alla crisi finale. Per sfuggire alla depressione, intraprende diversi viaggi; il primo verso il nord, sulle orme dell'amato Ibsen $^{11}$, il secondo verso sud, sino in Sicilia ${ }^{12}$.

Di questo grand tour ci è rimasto un taccuino di appunti filosofici e personali, in cui Weininger confessa: «Questo viaggio $\mathrm{mi}$ ha dato la consapevolezza di non essere un filosofo» ${ }^{13}$. All'amico Arthur Gerber scrive, manifestando la sua disperazione: «Attraverso un periodo molto brutto, brutto come mai in passato» ${ }^{14}$. Nello stesso periodo invia una lettera anche ad un altro amico fedele, Moritz Rappaport: «Va assai peggio di quanto credessi ancora due giorni fa. Non c'è quasi piú speranza» ${ }^{15}$.

Nel 1903, sulla base della sua tesi di laurea, intraprende la scrittura di Geschlecht und Characker, per dargli una veste meno letteraria e piú scientifica ${ }^{16}$. L'opera esce nel maggio 1903. Al suo ritorno a Vienna, chiede all'amico Gerber di aiutarlo a lavorare alla

\footnotetext{
${ }^{11}$ Visitò, nell'ordine, le città di Monaco, Norimberga, Bayreuth, Dresda, Crampas, Copenhagen, Cristiania.

${ }^{12}$ Weininger giungerà sino a Siracusa, passando per Roma, Napoli, Messina, Taormina, e Catania.

${ }^{13}$ O. Weininger, Taccuino e lettere, a cura di M. Cometa, Edizioni Studio Tesi, Pordenone 1986. Lettera a Arthur Gerber del 17 agosto 1902, Crampas, p. 57.

${ }^{14}$ Id., Lettera a Arthur Gerber del 5 agosto 1902, Norimberga, p. 48

${ }^{15}$ Id., Lettera a Moritz Rappaport (23-27 agosto 1903), Casamicciola, Isola d'Ischia, p. 77.

${ }^{16}$ Nel marzo 1903 riesce ad avere la disponibilità della pubblicazione dell'opera da parte dell'editore Braumüller ed il 29 maggio l'amico e corrispondente di Weininger, Arthur Gerber, ne riceve una copia.
} 
revisione del suo libro, in vista di una seconda edizione. Gerber, però, per piú giorni non riesce a trovare l'amico, che si nega puntualmente. Il 3 ottobre Weininger affitta la stanza in cui era morto l'amato Beethoven e al culmine della disperazione, pone fine alla sua esistenza, sparandosi dritto alla tempia. Muore, senza riprendere coscienza, il mattino seguente (4 ottobre), all'età di trentatré anni.

\section{Il caso Weininger: metafisica dell'amor sessuale e rapporto con la cultura mitteleuropea}

La celebrità di Weininger è in parte ascrivibile al successo di scandalo dovuto alla sua tragica morte. L'evento del suo suicidio diede inizio ad un vero e proprio caso, der Fall Weininger, come lo chiamerà J. Le Rider. Sulle pagine della rivista krausiana «Die Fackel» seguì un lungo e vivace dibattito, con interventi biografici del padre del filosofo suicida, ma anche con alcuni articoli di August Strindberg ${ }^{17}$, con il quale lo stesso Weininger era in contatto epistolare, dibattito che destò l'interesse persino di Ludwig Wittgenstein.

Un capitolo a parte meriterebbe la lunga e incredibile storia della fortuna di Weininger $^{18}$, delle numerose edizioni del suo scritto, dell'interesse per la sua misoginia metafisica, per la sua morfologia sessuale, interesse che si estese anche a pensatori come Oswald Spengler e Emil Cioran. Si parlò di "sparo nella nebbia" (Max Nordau), di

\footnotetext{
17 M. Cometa ha tradotto e pubblicato un breve scambio epistolare fra Weininger e Strindberg, Weininger-Strindberg. Lettere e testimonianze, in O. Weininger, Taccuino e lettere, cit., pp. 85-91.

${ }^{18}$ Sesso e carattere fu un vero e proprio Bestseller del tempo: ebbe dodici edizioni fra il 1903 e il 1910, altre diciotto fra il 1910 e il 1947. Fu tradotto in tutte le principali lingue europee, francese inglese e italiano, ma anche in polacco, in russo e in ebraico. Oswald Spengler citò Weininger nel suo Tramonto; ma anche altri interpreti del cosiddetto "nichilismo mistico", come lo ha chiamato Franco Volpi, si interessarono all'autore di Sesso e carattere, come l'apolide metafisico Cioran, che dichiara di essere attratto in Weininger da «le vertiginose esagerazioni, la smisuratezza della negazione, il rifiuto di ogni common sense, la feroce intransigenza, la ricerca permanente dell'assoluto, la mania di produrre un pensiero sino al punto in cui esso si sciolga da sé e distrugga l'edificio intero di cu esso stesso è parte» E. Cioran, Brief an Jacques Le Rider, in O. Weininger, Werk und Wirkung. I giornali tedeschi e francesi contemporanei di Weininger parlarono dello "sparo nella nebbia" (Max Nordau), ricollegando quello di Weininger ai casi delle cosí dette volontà malate di fin-de-siècle. In Italia, Giovanni Papini parlò di "suicidio metafisico", affiancandolo a quello di un vero Schopenhauer-Schüler, Philipp Mainländer. Anche in Italia, una numerosa schiera di intellettuali si interessò al caso Weininger: i fratelli de Chirico, poeti come Cardarelli e Saba, la scrittrice Sibilla Aleramo, il filosofo Julius Evola (che ne tradurrà l'opera principale), i letterati Ardengo Soffici e Italo Tavolato, lo psicologo Enrico Morselli, ma se ne occuperanno anche intellettuali illuminati come Giovanni Vailati, Piero Gobetti, Mario Calderoni, Delio Cantimori (responsabile della voce Weininger per la Treccani), Giovanni Amendola, Giovanni Boine, Scipio Slataper, Elias Canetti e Giuseppe Prezzolini. Quest'ultimo, in un primo momento, si era proposto di tradurre Sesso e carattere.
} 
"suicidio metafisico" (Papini) ${ }^{19}$, ricollegando quello di Weininger ai casi degli Schopenhauer-Schüler ${ }^{20}$ Carlo Michelstaedter e Philipp Mainländer ${ }^{21}$.

Tuttavia, va detto con chiarezza che quello di Weininger non fu un suicidio metafisico, come quello degli altri due schopenhaueriani testé citati, perché esso non fu causato da un motivo di origine filosofica. Si trattò invece di un suicidio commesso per disperazione e depressione, cioè per motivi esistenziali. Weininger, infatti, rifiutava l'idea della morte volontaria, in quanto la considerava un atto di viltà che negava la kantiana volontà buona. Tanto è vero che aveva scritto: «Il suicidio non è segno di coraggio, ma di viltà, sebbene esso sia di tutte le viltà la piú piccola» ${ }^{22}$. Non a caso, in Sesso e carattere aveva anche affermato che, in opposizione alla vita virile e morale dell'uomo, «La prova ultima e assoluta della completa nullità della vita femminile, della completa inesistenza in essa di un essere che la sovrasti, vien data dal modo in cui le donne si suicidano» ${ }^{23}$. E ancora, «L'uomo che si castiga mortificando il corpo [...] è vile quanto il suicida che si spara perché dispera di trionfare lui stesso» ${ }^{24}$. Ironia del fato, Weininger si suicidò sparandosi alla testa.

Entrando nello specifico della sua filosofia, Weininger presenta una metafisica che si proclama (erroneamente) immanentistica, fondata sulla legge dell'attrazione sessuale e sulla distinzione fra i tipi (idealtipi) di Uomo e di Donna. Proponendo una personalissima teoria del linguaggio, Weininger rileggeva il mondo in chiave simbolica, rifiutando da un lato la dialettica hegeliana e sostenendo, dall'altro, un radicale astoricismo dei valori.

\footnotetext{
${ }^{19}$ Giovanni Papini, «Il Resto del Carlino», 5 nov. 1910.

${ }^{20}$ Weininger fu presto accostato, in maniera erronea, a pensatori di ispirazione schopenhaueriana, come Michelstaedter e Mainländer, ma anche a Nietzsche. In Weininger, infatti, la cultura del tempo celebrava il promotore di una rivoluzione sessuale in senso maschilista, primato tristemente conteso dal suo meno noto contemporaneo Möbius, anch'egli in linea con lo schema ermeneutico già espresso dal predecessore J. Görres. Weininger veniva considerato come il promotore di una «igiene sociale», come il sostenitore dell'avvento degli uomini superiori, indebitamente tradotto nei termini nietzscheani di una "morale dei signori" e della pulizia razziale. Per esempio, Weininger fu per il circolo dei lacerbiani il filosofo metafisico in senso anticrociano, il filosofo della rivoluzione sessuale e dell'uomo di azione. Cfr. A. Cavaglion, La filosofia del pressappoco. Weininger, sesso, carattere e la cultura del Novecento. L'ancora del Mediterraneo, Napoli 2001.

${ }^{21}$ Per quanto riguarda la vicenda biografica e il pensiero di questo semisconosciuto seguace di Schopenhauer, del quale è in atto una vera e propria riscoperta, mi permetto di rinviare al mio Verso l'assoluto nulla. La Filosofia della redenzione di Philipp Mainländer, Pensa Multimedia, Lecce 2006.

${ }^{22}$ O. Weininger, Taccuino e lettere, cit. Lettera a Moritz Rappaport, Napoli (28 agosto) 1903, p. 79.

${ }^{23} \mathrm{GuCh}$, p. 365.

${ }^{24} \mathrm{GuCh}$, p. 423.
} 
La filosofia non è la storia, anzi della storia è proprio il contrario; non vi è filosofia che non abbia negato il tempo, non vi è filosofo per il quale il tempo sia stato una realtà come le altre $\operatorname{cose}^{25}$.

Il pensatore fonda la sua metafisica su di una simbolica universale ${ }^{26}$, sullo studio morfologico e fisiognomico della natura, ovverosia sull'analogia fra i viventi, avendo come modello quello caratterologico degli uomini ${ }^{27}$, già proposto da Schopenhauer ${ }^{28}$ e sviluppato poi dal suo discepolo Julius Bahnsen ${ }^{29}$. Per giustificare le sue tesi, Weininger aveva consultato una messe di fonti, da quelle biologiche e fisiche, a quelle mediche e psicologiche, a quelle filosofiche e letterarie, ordinandole in senso ascendente, ovvero dalle scienze della natura alla filosofia. Attraverso gli studi di G. Fechner e R. H. Lotze, Weininger si ricollegava alla Naturphilosophie di matrice schellinghiana, proponendo un'ermeneutica simbolica, come l'ha chiamata Michele Cometa ${ }^{30}$, ovvero individuando nell'Uomo e nella Donna i poli naturali rispetto i quali si innerva l'intera scala gerarchica della natura, che Weininger interpretava nel monistico di "anima del mondo". Ma la distinzione fra donna e uomo non è solo ontologica, bensì anche deontologica: all'uomo corrisponde la spiritualità, l'essere, l'attività e la moralità, là dove ex contrario alla donna corrisponde la sensualità, il non-essere, la passività, il peccato ${ }^{31}$. La donna può essere madre, rispetto alla specie, cioè come modello ideale dell'amore platonico; ma può essere

\footnotetext{
${ }^{25}$ Cfr. GuCh, p. 381.

${ }^{26}$ Cfr. O. Weininger, Metafisica (L’idea di una "simbolica” universale, psicologia animale, con una psicologia del "delinquente” piuttosto esaustiva, ecc) in Id., Delle cose ultime, tr. it. di F. Cicoira, intr. di A. Cavaglion, Edizione Studio Tesi, Pordenone 1992, pp. 169-198.

${ }^{27}$ O. Weininger, Contributo alla caratterologia (Cercatori e sacerdoti, su Friedrich Schiller, frammenti su Richard Wagner e il "Parsifal”), Delle cose ultime, cit., pp. 117-140.

${ }^{28} \mathrm{Su}$ questo tema si leggano le recenti osservazioni di G. Gurisatti, Caratterologia, metafisica e saggezza. Lettura fisiognomica di Schopenhauer, presentazione di F. Volpi, Il Poligrafo, Padova 2002.

${ }^{29} \mathrm{Si}$ ricordi che J. Bahnsen aveva intitolato cosí una sua voluminosa opera Beiträge zur Charakterologie. Mit besonderer Berücksichtigung pädagogischer Fragen (2 Bde., Leipzig 1867) e che Weininger dimostra di conosce Bahnsen (Delle ultime cose, cit., p. 11), riprendendone probabilmente il titolo nella sua opera. Su Bahnsen, cfr. il prezioso lavoro di ricostruzione filosofico-storiografica di Domenico M. Fazio, La scuola di Schopenhauer, in La scuola di Schopenhauer. Testi e contesti, a cura del Centro interdipartimentale di ricerca su Arthur Schopenhauer e la sua scuola, Pensa Multimedia, Lecce 2009, pp. 13-212:73-93.

${ }^{30}$ M. Cometa, Anatomia degli angeli. Otto Weininger e la simbolica, in O. Weininger, Taccuino e lettere, cit., pp. VII-XXX, in particolare p. XIX.

${ }^{31} \mathrm{Cfr}$. in particolare GuCh, cap. XII «L'essenza della donna e il suo senso nell'universo», pp. 328-385, in cui Weininger scrive apertis verbis che «La donna è alogica e amorale» e «La donna, ed anche la donna nell'uomo, è il simbolo del nulla» (p. 378), «Il senso della donna consiste dunque nel suo essere il non-senso» (p. 379), «La donna è la colpa dell'uomo» (p. 384). Queste valutazioni fanno tutt'uno con la misoginia sessuale weiningeriana, in base alla quale Weininger aveva sostenuto che «l'uomo possiede il pene, mentre la donna è posseduta dalla vagina», Sessualità maschile e sessualità femminile, parte II, cap. II, p. 135.
} 
anche etèra, rispetto all'individuo ${ }^{32}$, cioè come causa dell'appetito sessuale. In ogni caso, la sua natura è legata alla sessualità, alla caduta nel peccato.

Con ciò, Weininger riprende il modello fechneriano dell'Allbesseltheit [polarità naturale], dotandolo però di proprie categorie morali e religiose. Inoltre, se il naturalismo di Fechner giunge a Weininger come utile strumento per distinguere, nell'unità dell'universo, una differenziazione di gradi dell'essere, la filosofia morale kantiana invece fornisce all'universo weiningeriano l'unità di misura del dovere, in base alla quale dividere il bene dal male, l'essere puro dall'essere contaminato, l'io intelligibile da quello empirico:

Questa [l'etica] vuole che l'Io intelligibile agisca, libero da tutte le scorie dell'Io empirico, per cui solo attraverso l'etica può essere realizzato pienamente e nella sua purità quell'essere, che già la logica ci annuncia nella forma allettatrice di una realtà che già sarebbe presente ${ }^{33}$.

La questione fondamentale, quindi, per il filosofo austriaco è quella morale. La metafisica ha come fondamento l'etica. Weininger asserisce di partire dal primato della ragion pratica di $\mathrm{Kant}^{34}$. Nei fatti, Weininger ha già riletto la ragion pratica kantiana alla luce della filosofia del dovere di Fichte ${ }^{35}$, per affermare un primato della morale che l'autore di Sesso e carattere cerca di dimostrare sulla base della legge dell'attrazione sessualità, ovvero sulla diversità dei tipi sessuali. I tipi sessuali sono idee-limite, non esistenti nella realtà, che assolvono solamente ad una funzione ad un tempo euristica ed epistemologica. Per Weininger, infatti, non esistono l'uomo e la donna in senso puro, ma esclusivamente una varietà di gradi intermedi fra l'uno e l'altra. L'uomo cerca nella donna il mascolino che è in lei, per ricomporre il modello ideale dell'uomo perfetto. Diversamente da Schopenhauer $^{36}$, per Weininger l'attrazione non si fonda sulla platonica complementarità dei sessi rispetto alla specie, non si raggiunge cioè un essere completo androgino. Il pensatore viennese dichiara invece che l'istinto sessuale è alimentato dalla ricerca dell'identità del tipo sessuale nell'altro. Si tratta, cioè, di una sorta di androcentrismo

\footnotetext{
${ }^{32}$ Cfr. GuCh, cap. X «Maternità e prostituzione», pp. 269-305.

${ }^{33} \mathrm{GuCh}$, cap. VIII «La logica, l'etica, l'Io», p. 210.

${ }^{34} \mathrm{GuCh}$, cap. VIII «La logica, l'etica, l’Io», p. 208: «Per quel che riguarda l'etica, il presente studio si rifà completamente alla filosofia morale di Kant».

${ }^{35}$ Nel pensiero di Fichte Weininger vede, fra l'altro, il ponte di unione fra logica ed etica, GuCh, p. 209: «Logica ed etica sono però, in ultima istanza, una sola e medesima cosa: un dovere verso se stessi. [...] L'etica è possibile solo secondo le leggi della logica, cosí come la logica è in pari tempo legge etica».

${ }^{36}$ Sul tema, mi permetto di rinviare a F. Ciracì, Schopenhauer, Weininger e l'amor sessuale, La passione della conoscenza. Studi in onore di Sossio Giametta, a cura del Centro interdipartimentale di ricerca su Arthur Schopenhauer e la sua scuola dell'Università del Salento, Pensa MultiMedia, Lecce 2010, pp. 117-148.
} 
sessuale identitario, in cui il mascolino ricerca il mascolino nella persona desiderata, per ricostituire l'idea dell'uomo puro perfetto.

Rispetto ai pensatori ai quali spesso lo si associa, Schopenhauer e Nietzsche, Weininger risulta essere distante in maniera siderale per "visione del mondo". Egli non è né un ateo come Schopenhauer, né un nichilista come Nietzsche. Weininger, infatti, rifiuta sia l'impianto metafisico sia il pessimismo antropologico della volontà di Schopenhauer, cosí come nega il relativismo dei valori (che posseggono invece, in quanto tali, un'evidenza logica) e il prospettivismo morale di Nietzsche. «Ogni volontà - aveva scritto il filosofo viennese - è volontà di valore, ed ogni istinto è rivolto al piacere; non esiste una volontà di piacere e nemmeno una volontà di potenza....» ${ }^{37}$.

Per Weininger le azioni si svolgono nell'ambito di una temporalità lineare ed irreversibile $^{38}$, che è condizione stessa della moralità dell'agire umano, proprio in opposizione al filosofema dell'eterno ritorno nietzscheano, che liberava l'uomo da ogni finalismo e riconsegnava all'azione umana il suo significato secolare ${ }^{39}$. Contrariamente a Nietzsche, per il quale la morale è un prodotto storico umano, troppo umano, il senso della vita per Weininger è mistico. È possibile cioè una unio mystica con Dio nel mondo intelligibile, attraverso l'azione morale. Quello weiningeriano, quindi, è un pessimismo mistico, edulcorato dalla prospettiva escatologica ${ }^{40}$ : all'uomo, nel senso di genere sessuale, è data quindi una via di uscita dalla caduta nella temporalità, una via di redenzione individuale definitiva. Redenzione finale totale, che anche la metafisica pessimistica di Schopenhauer aveva negato, indicando come vie di liberazione dalla volontà l'arte, intesa come effimera sospensione estetico-estatica, e la noluntas, come via ascetica del nirvana, con il quale si giunge per gradi solo ad un nihil negativum dell'individuo rispetto al mondo. Anche il preteso eroismo di Weininger non è quello nietzscheano dello Zarathustra, non si tratta cioè di un'accettazione tragica della fine di tutti i valori assoluti,

\footnotetext{
${ }^{37} \mathrm{GuCh}$, p. 360.

${ }^{38}$ Cfr. O. Weininger, Sull'irreversibilità del tempo (il suo significato etico, con l'aggiunta di considerazioni su tempo, spazio e volontà in generale) in Id., Delle cose ultime, cit., pp. 143-165.

${ }^{39} \mathrm{Il}$ tema dell'irreversibilità del tempo di Weininger, fra l'altro, avrebbe affascinato anche i fratelli Giorgio e Andrea de Chirico (Alberto Savinio), che ne avrebbero tratto ispirazioni per la loro "pittura metafisica".

${ }^{40} \mathrm{O}$. Weininger, "Peer Gynt" e Ibsen. (Note sull'erotismo, sull'odio e l'amore, sul delitto, sulle idee di padre e di figlio) in Delle cose ultime, cit., p. 37: «Vi sono allora due possibilità: o l'uomo è pessimista per natura e non crede mai veramente alla redenzione, bensì all'eterna discordia e alla dannazione su questa terra; è il tipo dell'uomo, a livelli piú volgari, aspro, maligno, incline ad imprecare. Oppure viene al mondo recando già in sé la fede nella redenzione, è deciso a raggiungerla, vale a dire che è capace di conseguirla già in terra. È l'uomo mite, benevolo, che non ama criticare aspramente».
} 
dio non è morto, ma la divinità vive e trascende il mondo reale, determinandone il fine ultimo.

In conclusione, anche se si farà battezzare con rito protestante, e pur avendo clamorosamente rinnegato la propria origine e la propria religione ebraica, de facto Weininger dimostra di essere rimasto all'interno della sua tradizione, di averla rielaborata ad uso proprio. L'afflato messianico e la tensione mistico-religiosa sono rivisitate alla luce della metafisica del sesso, dell'antico mito protoromantico dell'androgino. Con Fechner, Weininger non esita nemmeno a interpretare il pensiero di Kant in senso mistico:

L'idea di un essere perfettamente libero è l'idea di Dio; l'idea di un essere misto di libertà e di non-libertà e l'idea di uomo. Nella misura in cui l'uomo è libero, cioè vuole liberamente, egli è Dio. Cosí l'etica kantiana è, nel suo fondo, mistica ${ }^{41}$.

Inoltre, la sua lettura in chiave analogico-simbolica del mondo sembra ripercorre l'antica tradizione kabbalistica, e si ricollega ad un tempo al misticismo di Jakob Böhme e soprattutto a quello di Baader, il cosiddetto mago del Sud, che Weininger cita e ha presente in Sesso e carattere ${ }^{42}$.

Se Svevo aveva introdotto nei suoi personaggi la distinzione fra lottatori e contemplatori, propendendo decisamente con Schopenhauer per i primi, per Weininger, che si distinse sempre per vocazione profetica, il mondo del pensiero doveva dividersi in sacerdoti e ricercatori. Il nostro pensatore, che pur si considerava un ricercatore dello spirito, in realtà appartenne alla schiera dei sacerdoti. Si considerava e si proclamava infatti il «fondatore dell'unica scienza medica veramente efficace, dell'unica vera patologia, nonostante lo scarso numero di riconoscimenti positivi» ${ }^{43}$.

Egli fu un profeta, dunque, proveniente da quella cultura mitteleuropea della crisi, visse in maniera radicale l'esperienza di quel disagio esistenziale della sua stessa generazione, offrendo una soluzione che portava lo stigma della rivoluzione psicoanalitica freudiana e del nuovo linguaggio analogico-simbolico dei viennesi. Tuttavia, egli rimase distante dallo «sguardo orrificato verso un mondo sdivinizzato» ${ }^{44}$ di Schopenhauer, distante

\footnotetext{
${ }^{41} \mathrm{GuCh}$, p. 361.

${ }^{42}$ Cfr. F. Ciracì, Schopenhauer, Weininger e l'amor sessuale, cit., pp. 138-145.

${ }^{43}$ O. Weininger, Taccuino e lettere, cit., pp. 5-6.

${ }^{44}$ Fr. Nietzsche, La gaia scienza, tr. it. di S. Giametta, UTET, Milano 2000, aforisma 357, pp. 334-339: p. 339.
} 
dalla critica illuminata contro la morale del filosofo dello Zarathustra, distante anche dall'analisi esistenziale e dalle innovazioni concettuali di grandi maestri dello spirito e della Krisis, che al pensiero di Schopenhauer e di Nietzsche avevano attinto, come Carlo Michelstaedter, Italo Svevo e Robert Musil.

\section{Referências}

WEININGER, O. Sesso e carattere. Tr. it. di J. Evola, intr. di F. Antonini, coll. Orizzonti dello spirito. Roma: Edizioni Mediterranee, 1992.

. Taccuino e lettere, a cura di M. Cometa. Pordenone: Edizioni Studio Tesi, 1986.

- Metafisica (L’idea di una "simbolica" universale, psicologia animale, con una psicologia del "delinquente" piuttosto esaustiva, ecc) in Id., Delle cose ultime, tr. it. di F. Cicoira, intr. di A. Cavaglion. Pordenone: Edizione Studio Tesi, 1992.

CAMERINO, A. G. A mo' di conclusione. Lo specifico mitteleuropeo e i maggiori scrittori giuliani del Novecento, in Id., La persuasione e i simboli: Michelstaedter e Slataper. Napoli: Liguori, 2005.

CAVAGLION, A. La filosofia del pressappoco. Weininger, sesso, carattere e la cultura del Novecento. Napoli: L’ancora del Mediterraneo, 2001.

CIRACÌ, F. Verso l'assoluto nulla. La Filosofia della redenzione di Philipp Mainländer. Lecce: Pensa Multimedia, 2006.

Schopenhauer, Weininger e l'amor sessuale, La passione della conoscenza. Studi in onore di Sossio Giametta. A cura del Centro interdipartimentale di ricerca su Arthur Schopenhauer e la sua scuola dell'Università del Salento. Lecce: Pensa MultiMedia, 2010.

FAZIO, D. M. La scuola di Schopenhauer. In: La scuola di Schopenhauer. Testi e contesti. A cura del Centro interdipartimentale di ricerca su Arthur Schopenhauer e la sua scuola. Lecce: Pensa Multimedia, 2009.

GURISATTI, G. Caratterologia, metafisica e saggezza. Lettura fisiognomica di Schopenhauer. Presentazione di F. Volpi. Padova: Il Poligrafo, 2002.

HELLENBERGER, H. F. The Discovery of the Unconscious. The History and Evolution of Dynamic Psychiatry. New York: Basic Books, 1970, tr. it. Id., La scoperta dell'inconscio. Storia della psichiatria dinamica, 2 vol., Torino: Bollati Boringhieri, 2001.

JANIK, A.; TOULMIN, S. La grande Vienna. Nella Vienna di Schönberg, di Musil, di Kokoschka e del dottor Freud, la formazione intellettuale del grande filosofo Wittgenstein. Garzanti, 1975.

LESSING, Th. Der jüdische Selbsthaß (1930). Mit einem Essay von Boris Groys. Berlin: Nachdr. der Ausg. Matthes \& Seitz, 2004. 
Revista Voluntas: estudos sobre Schopenhauer - $1^{\circ}$ semestre 2011 - Vol. 2 - No 1 - ISSN: 2179-3786 - pp. 95-107.

Recebido: 03/02/11

Received: 02/03/11

Aprovado: 22/05/11

Approved: 05/22/11 